University of Wollongong

Research Online

Faculty of Engineering and Information

Faculty of Engineering and Information

Sciences - Papers: Part A

Sciences

2012

Dynamic impression insole in rheumatoid foot with metatarsal pain

Bao-Chi Chang

National Yang Ming University

Jia-Yi Wang

Taipei Medical University

Biing-Shiun Huang

National Yang Ming University

Hsiao-Yi Lin

National Yang Ming University

Winson Lee

University of Wollongong, ccwlee@uow.edu.au

Follow this and additional works at: https://ro.uow.edu.au/eispapers

Part of the Engineering Commons, and the Science and Technology Studies Commons

Research Online is the open access institutional repository for the University of Wollongong. For further information contact the UOW Library: research-pubs@uow.edu.au 


\title{
Dynamic impression insole in rheumatoid foot with metatarsal pain
}

\author{
Abstract \\ Background \\ Custom molded insoles with metatarsal supports are used to redistribute excessive loading under the \\ metatarsal heads in patients with metatarsalgia. However, these pressure reductions are usually \\ insufficient for the rheumatoid foot with painful deformed metatarsal heads. We developed an effective \\ insole made by sequential foam padding under successive walking impression.
}

\section{Methods}

Seventeen consecutive rheumatoid arthritic outpatients with metatarsal pain participated in this repeated measures study of 7-mm flat Ethylene Vinyl Acetate, custom molded and dynamic impression insoles. Peak plantar pressure, pressure-time integral, contact area and mean force were measured by a Pedar- $X$ mobile system. Pain levels were assessed using a Visual Analog Scale (0-10).

\section{Findings}

Compared to the Ethylene Vinyl Acetate control, the metatarsal head peak pressure and pressure-time integral were significantly reduced in dynamic impression insoles by $46.3 \%(P<0.001)$ and $48.9 \%(P<$ 0.001 ), respectively. Compared to the custom molded insole, the dynamic impression insole significantly reduced $18.3 \%$ of peak pressure $(P<0.001)$ and $20.1 \%$ of pressure-time integral $(P<0.001)$ by increasing $8.1 \%$ of contact area $(P=0.005)$ at the metatarsal heads, but there were no significant differences in all variables at the heel. After using the dynamic impression insole, the mean pain score was significantly reduced from 7.6 to $1.1(P<0.001)$, and six participants experienced total pain-relief in walking.

\section{Interpretation}

Dynamic impression insoles effectively relieve metatarsal pain because of a larger weight-bearing area. Forefoot shape during walking should be taken into consideration in orthotic designs for maximum pressure reduction. Consequently, we recommend using materials with memory properties to dynamically accommodate painful metatarsal heads.

\section{Keywords}

dynamic, metatarsal, foot, rheumatoid, insole, impression, pain

\section{Disciplines}

Engineering | Science and Technology Studies

\section{Publication Details}

Chang, B., Wang, J., Huang, B., Lin, H. \& Lee, W. C. C. (2012). Dynamic impression insole in rheumatoid foot with metatarsal pain. Clinical Biomechanics, 27 (2), 196-201. 

pain. Clinical Biomechanics. 2012, 27, 196-201.

\section{Dynamic impression insole in rheumatoid foot with metatarsal pain}

${ }^{\mathrm{b}}$ National Yang-Ming University, School of Medicine, Taiwan, R.O.C.

$14{ }^{\mathrm{c}}$ Graduate Institute of Medical Sciences, College of Medicine, Taipei Medical University, Taipei, 15 Taiwan, R.O.C.

$16{ }^{\mathrm{d}}$ Division of Allergy, Immunology and Rheumatology, Department of Medicine, Taipei Veterans

17 General Hospital, Taiwan, R.O.C.

18 e Department of Health Technology and Informatics, The Hong Kong Polytechnic University, Hong

19 Kong

$20{ }^{\dagger}$ Equal contributions

21 Running title: Dynamic Impression Insole for Rheumatoid Arthritis

$23 *$ Corresponding author

24 Dr. Winson Lee 
1 Department of Health Technology and Informatics

2 The Hong Kong Polytechnic University

3 Email: htwinson@inet.polyu.edu.hk

$4 \quad$ Tel: +852 27664942

$5 \quad$ Fax: +852 23624365

6 


\section{Abstract}

2 Background. Custom molded insoles with metatarsal supports are used to redistribute excessive

3 loading under the metatarsal heads in patients with metatarsalgia. However, the pressure reductions

4 are usually insufficient for rheumatoid feet with painful deformed metatarsal heads. We developed

5 an effective insole made by sequential padding of foams with different compressibility under

6 successive walking impression.

7 Methods. Seventeen consecutive rheumatoid arthritic outpatients with metatarsal pain participated

8 in this repeated measures study of 7-mm flat Ethylene Vinyl Acetate, custom molded and dynamic

9 impression insoles. Peak plantar pressure, pressure-time integral, contact area and mean force were

10 measured by Pedar-X mobile system. Pain levels were assessed using a Visual Analog Scale (0-10).

11 Findings. Compared to the Ethylene Vinyl Acetate control, the metatarsal heads peak pressure and

12 pressure-time integral were significantly reduced in dynamic impression insoles by $46.3 \%(P<0.001)$

13 and 48.9\% $(P<0.001)$, respectively. Compared to the custom molded insole, the dynamic impression

14 insole significantly reduced $18.3 \%$ of peak pressure $(P<0.001)$ and $20.1 \%$ of pressure-time integral

$15(P<0.001)$ by increasing $8.1 \%$ of contact area $(P=0.005)$ at the metatarsal heads, but no significant

16 differences in all pressure-related variables at the heel. After using the dynamic impression insole,

17 the pain score was significantly reduced from 7.6 to $1.1(P<0.001)$, and six participants experienced

18 total pain-relief under the metatarsal heads in walking.

19 Interpretation. Because of a larger weight-bearing area, dynamic impression insoles are effective

20 to relieve metatarsal pain. Forefoot shapes during walking should be taken into considerations in

21 orthotic designs for maximum pressure reduction. Consequently, we recommend using the materials

22 with memory properties to dynamically accommodate the painful metatarsal heads.

24 Key words: rheumatoid arthritis; metatarsalgia; pressure measurement; insole. 
1. Introduction

3 The prevalence of rheumatoid arthritis (RA) is approximately $0.8 \%$, ranging from $0.3 \%$ to $2.1 \%$ of

4 the population (Fauci et al., 2008). Involvement of metatarsophalangeal joints has been reported to

5 be as high as $80 \%$ to $92 \%$ during the course of the disease (Vainio et al., 1975; Speigel et al., 1982).

6 The most common forefoot deformities are hallux valgus and claw/hammer toes with dorsal

7 subluxation or dislocation of the lesser metatarsophalangeal joints (Jeng et al., 2008). Toe

8 deformities may cause prominence of the metatarsal heads (MTH) and distal displacement of fat-pad

9 cushion beneath the MTH, resulting in the metatarsal pain in RA patients (Sumpio, 2000). Foot pain

10 frequently leads to limitation of activities of daily life and deterioration of life quality (Kerry et al.,

11 1994; van der Leeden et al., 2006).

12 Plantar pressure analyses have been widely applied to biomechanical research and orthotic

13 evaluation in rheumatoid foot (Otter et al., 2004; Hodge et al., 1999). In RA with forefoot pain,

14 metatarsophalangeal subluxation and erosion have been related to increased pressure under the

15 forefoot (van der Leeden et al., 2006). Even though RA patients with metatarsal pain may not have

16 particularly high peak pressure, a decrease in plantar pressure still reduces subjective ratings of pain

17 in their walking (Hodge et al., 1999). Foot orthoses have been commonly used in clinical practice to

18 reduce plantar pressure and subsequent pain in management of rheumatoid feet (Clark et al., 2006).

19 The main strategies are to shift the excessive MTH forces by custom insoles with a metatarsal dome

20 or a metatarsal bar placed proximal to MTH (Postema et al., 1998; Hodge et al., 1999; Chalmers et

21 al., 2000; Jackson et al., 2004). Nevertheless, both types of metatarsal pads need to be appropriately

22 positioned to achieve their effectiveness (Clark et al, 2004). Previous studies of RA with

23 metatarsalgia (Hodge et al., 1999; Jackson et al., 2004) reported that the metatarsal padding provides

$24 \quad 11.3 \%$ to $21.8 \%$ peak pressure reduction at the MTH. However, the force redistribution with the use 
1 of metatarsal support may cause a discomfort in the area proximal to $\mathrm{MTH}$, affecting patient

2 acceptance (Clark et al, 2004). Therefore, the therapeutic efficacies of custom molded insoles with a

3 metatarsal support vary widely with their designs and materials for the rheumatoid foot with

4 metatarsalgia (Conrad et al., 1996; MacSween et al., 1999; Novak et al., 2009).

5 A Plastazote foam is generally used in neuropathic foot because of the excellent self-molding

6 properties and therapeutic effectiveness, but it has a very short life due to high compressibility

7 (Bertram et al., 1997). The Plastazote combined with less compressible foam Aliplast has been

8 reported to relatively increase its longevity, but it still limits the effective use within a few months

9 (Bertram et al., 1997). Furthermore, an insufficient toe-box space of shoes would have restricted the

10 initial thickness for sufficient pressure reduction. We designed a simple and effective method that a

11 dynamic impression insole was made by sequential padding of foams with different compressibility

12 under successive dynamic impression in daily walking. Very few studies have provided the plantar

13 pressure analysis in the accommodative insole for the RA subjects with metatarsalgia. The purposes

14 of this study were to investigate the biomechanics of dynamic impression insole in plantar pressure

15 reduction, as well as to compare with custom molded insole among seventeen RA adults with

16 metatarsal pain.

\section{Methods}

\section{2.1. Participants}

20 Nineteen consecutive RA patients with metatarsal pain, who were diagnosed by rheumatologists

21 according to American College of Rheumatology criteria (Arnett et al., 1988) were recruited from

22 the podiatry outpatient clinic of Taipei Veteran General Hospital. Seventeen participants (15 females

23 and 2 males, 16 bilateral feet and 1 unilateral foot involvements) completed this study without

24 interruption of using dynamic impression insoles. One of the dropouts was due to an insufficient 
1 toe-box space for the severely deformed forefoot with the Plastazote in walking, and the other was

2 due to difficulty following the schedule required by this study. The participants were active in

3 walking without any aids for plantar pressure measurement. There were no flexible flat feet in all

4 participants. The demographic characteristics of participants are given in table 1 . The locations (\%

5 morbidity) of metatarsal pain were the first MTH (8.8\%), the second MTH (76.5\%), the third MTH

6 (70.6\%), the fourth MTH (20.6\%), and the fifth MTH (2.9\%). The metatarsal pain was complicated

7 with toe deformities including hallux valgus (76.5\%) and claw/hammer toes (58.8\%). All subjects

8 were instructed to wear extra-width and extra-depth shoes that could accommodate deformed

9 forefoot and minimal 10-mm thick insole. This study was approved by the Ethics Committee of the

10 Taipei Veterans General Hospital, and informed consent was obtained from all participants.

\section{2.2. Dynamic impression insole}

12 The procedures of fabricating the dynamic impression insole were divided into four steps in four 13 visits to the podiatry clinic.

14 1) A 9-mm thick Plastazote (15 Shore A hardness, Schein orthopädie service KG. Remscheid,

15 Germany) was inserted into wide extra-depth shoes in each RA participant. The Plastazote in the

16 MTH region would be compressed more than one half of the original thickness about 2-3 weeks, 17 depending on the level of walking activity.

18 2) The Plastazote in the toes area was fattened to the same thickness as the deepest toe impression. A

19 6.5-mm thick P-cell (21 Shore A hardness, Acor orthopaedic Inc. Cleveland, Ohio, USA) was

20 adhered to the bottom of the impressed Plastazote with double-sided adhesive tape. The P-cell in

21 the forefoot region was ground to the thickness that patient's forefoot could accommodate.

22 3) After walking compression for about 2-3 weeks, a piece of metatarsal pad and arch support made

23 of Ethylene Vinyl Acetate (EVA) of 40 Shore A hardness was attached to the bottom of impressed

24 insole just proximal to the first, second, and third MTH according to the foot impression. 
1 4) After further walking for about 2-3 weeks, a 2-mm thick Multiform (30 Shore A hardness, Schein

2 orthopädie service KG. Remscheid, Germany) was adhered to the top of the impressed insoles.

3 The EVA was also used to adjust unequal thickness between medial and lateral side (figure 1).

$4 \quad$ 2.3. Custom molded insole

5 1) Foot impression was taken in an impression box while holding the subtalar joint at a neutral

6 position as possible.

7 2) A 3-mm Multiform was used as a top layer and cork as a bottom layer. The middle layer was

8 P-cell at the forefoot, EVA at the midfoot and hindfoot. A metatarsal support was incorporated

$9 \quad 5$-mm proximal to all MTH in the cork layer (figure 1).

10 3) All layers were sequentially added on the positive plaster cast in vacuum former after gluing and

11 heating in the oven.

12 2.4. Evaluation of the insoles

13 The pain levels of using the dynamic impression insoles at different stages were recorded from 0

14 (no pain) to 10 (the worst pain) according to the Visual Analog Scales (VAS) pain score. Pain levels

15 were assessed on the first visit (before treatment with insoles), on the second visit (2-3 weeks after

16 using the 9-mm thick Plastazote), and on the day for the plantar pressure measurements (1 month

17 after the final step of fabricating the dynamic impression insole). The custom molded insoles were

18 prescribed to the participants at their second visits. The pain levels of using the custom molded

19 insole were recorded on the day for the plantar pressure measurements. The participants had about

20 half day of time of using the custom molded insoles before assessing the pain levels and plantar

21 pressure. Plantar pressure analysis was performed using the Pedar-X mobile in-shoe system (Novel

22 gmbh, Munich, Germany). Three kinds of insoles including 7-mm flat EVA control (40 Shore A

23 hardness), dynamic impression insole and custom molded insole were randomized in the plantar 
1 pressure measurements. The measurements were carried out under a comfortable and stable walking

2 speed preferred by the participants one month after the dynamic impression insoles were fabricated.

3 The Pedar system was calibrated according to the manufacturer's instructions before plantar pressure

4 assessment. Plantar pressures were measured at a frequency of $100 \mathrm{~Hz}$ in the middle 5-meter path of 5 an 8-meter walkway. All participants walked 5 laps without any walking aids for plantar pressure 6 measurements on the same day. Since the walking velocity has been demonstrated to influence 7 plantar pressure (Zhu et al., 1995; Kang et al., 2008), walking trials would be adjusted and repeated 8 if the speed was out of $5 \%$ from the mean velocity of three different insoles. All pressure data were 9 processed with the Novel-Win Multimask analysis software (Novel gmbh, Munich, Germany). The plantar area was divided into 4 regions that were heel (0-30\% of foot length), midfoot (30-60\% of

11 foot length), metatarsal heads (60-85\% of foot length), and toes (85-100\% of foot length). Only the 12 left foot was chosen for statistic analysis due to the requirement of data independence.

\section{2.5. Statistical analysis}

14 The required sample size was estimated by GPower 3.1.2 (Faul et al., 2007), assuming a power of 0.8 and an alpha level of 0.05 . From the data of previous study in RA with metatarsalgia (Jackson et

17 difference of $15 \%$ in peak plantar pressure between two dependent means. All statistical analyses

18 were performed using SPSS 17.0 (SPSS Inc, Chicago, Illinois). One-way ANOVA with repeated

19 measures was undertaken to determine significant differences in peak pressure, pressure-time

20 integral, contact area and mean force among the three insoles, and post-hoc Bonferroni test to

21 perform pair-wise comparisons among the three insoles. Two-tailed paired t-test was used to

22 compare the VAS pain scores before and after treatment with insoles. The differences were

23 considered significant if $P<0.05$. 


\section{3. Results}

2 After using the 9-mm thick Plastazote with the extra-width and extra-depth shoes, the mean VAS

3 pain score was significantly decreased from 7.6 (range 5-10, SD=1.7) to 4.1 (range 2-6, SD=1.2) in

4 daily walking $(P<0.001)$. One month after the dynamic impression insoles were well fabricated, the

5 mean VAS pain score was further significantly decreased to 1.1 (range $0-4$, SD=1.1, $<<0.001$ ), and 6

6 of 17 participants experienced no pain under the MTH in their walking. In addition, all participants

7 did not feel any discomfort or pain under the region proximal to MTH when using dynamic

8 impression insoles. The pain score (mean=2.5, range $0-6, \mathrm{SD}=1.7$ ) with the use of custom molded

9 insole was significantly higher than that of dynamic impression insole $(\mathrm{p}<0.001)$. Fourteen

10 participants preferred using the dynamic impression insoles, but only three participants considered

11 no differences between two insoles. The mean walking speed in the pressure measurement was 57.7

$12 \mathrm{~m} / \mathrm{min}$ (range 42.9-89.3, $\mathrm{SD}=12.1 \mathrm{~m} / \mathrm{min}$ ), which was slower than mean normal walking speed of 77

$13 \mathrm{~m} / \mathrm{min}$ in people of similar age group (Kang et al., 2008).

14 At the MTH, one-way ANOVA with repeated measures revealed significant differences among the

15 three insole in peak pressure $(P<0.001$, table 2$)$, pressure-time integral $(P<0.001$, table 3$)$, mean

16 force $(P<0.001$, table 4$)$ and contact area $(P<0.001$, table 5). Compared to the 7 -mm flat EVA

17 control, the peak pressure and pressure-time integral were reduced by $46.3 \%(P<0.001)$ and $48.9 \%$

$18(P<0.001)$ along with an $18.1 \%$ reduction in mean force $(P<0.001)$ and a $15.1 \%$ increase in contact

19 area $(P=0.002)$ with statistical significances in the dynamic impression insole. Compared to the

20 custom molded insole, the dynamic impression insole provided an $18.3 \%$ reduction in peak pressure

$21(P<0.001)$, a $20.1 \%$ reduction in pressure-time integral $(P<0.001)$, an $8.1 \%$ increase in contact area

$22(P=0.005)$ with significance, but no significant difference was found in mean force $(P=1.000)$.

23 At the midfoot, ANOVA revealed significant differences in mean force $(P<0.001)$ and contact

24 area $(P<0.001)$ among three insoles, but no significant differences in peak pressure $(P=0.097)$ and 
1 pressure-time integral $(P=0.909)$. Compared to the EVA control, the dynamic impression insole

2 significantly increased the mean force by 2.6 times $(P<0.001)$ and the contact area by 2.0 time $(P$

$3<0.001$ ), while the custom molded insole significantly increased the mean force by 2.1 times ( $P$

$4<0.001)$ and the contact area by 1.9 times $(P<0.001)$. The dynamic impression insole had

5 significantly greater mean force than custom molded insole ( $P=0.004)$, but no significant difference

6 in contact area $(P=0.130)$.

$7 \quad$ Repeated measures ANOVA also showed significant differences among three insoles in four

8 pressure-related variables at the heel (all $P<0.001$, table 2 to 5). However, post-hoc Bonferroni test

9 revealed no significant differences between dynamic impression insole and custom molded insole in 10 peak pressure $(P=0.124)$, pressure-time integral $(P=0.999)$, contact area $(P=0.058)$ and mean force $11 \quad(P=1.000)$.

12 In pair-wise comparisons between the three insoles at the MTH, the post-hoc power analysis of 13 peak pressure revealed statistical powers from 0.81 to1, which were larger than 0.8 of priori power 14 analysis.

\section{Discussion}

17 Rheumatoid arthritis is three times more common in females than in males (Fauci et al., 2008).

18 Furthermore, metatarsal pain frequently occurs in women who like to wear fashion shoes with

19 restricted toe-box. These could account for the fact that most of our participants were females in

20 gender distribution. The RA participants with metatarsal pain had very high morbidity of hallux

21 valgus and claw/hammer toes in this study. Most participants were bilateral feet involvements

22 including metatarsal pain, which was consistent with clinical manifestation of symmetric arthritis in

23 the RA. The mean VAS pain score after using 9-mm Plastazote in walking was decreased to 4.1,

24 which was very close to a similar study (Chalmers et al., 2000). The walking pain score with the use 
1 of the dynamic impression insole was lower than that with the custom molded insole. It was hard to

2 ensure that each participant used the same amount of time in using the custom molded insoles and

3 the dynamic impression insoles. However, the pain score with the use of our custom molded insole

4 was comparable to those reported in previous studies (Postema et al., 1998; Hodge et al., 1999). The

5 participants were given about half day of time of getting accustomed to the custom molded insoles,

6 before assessing the pain levels and plantar pressure.

8 In dynamic impression insole, both mean VAS pain score and peak pressure were further reduced

9 after sequential padding and successive walking compression. Approximate one third of the

10 participants experienced total pain-relief under MTH in their daily walking. Compared to the custom

11 molded insole, the dynamic impression insole apparently reduced the peak pressure, pressure-time

12 integral and increased the contact area at the MTH. However, there was no significant difference in

13 mean force at the area. Since both the dynamic impression and custom molded insoles have been

14 incorporated in the metatarsal region with similar metatarsal supports and the same P-cell material

15 (figure 1), their comparable force-shifting and force-absorbing effects could account for no

16 significant differences in mean force. Therefore, the contact area is a main determining factor that

17 causes the different effectiveness between two insoles in reducing peak pressure and pressure-time

18 interval at the MTH. Although the MTH peak pressure was reduced by $34.4 \%$ after incorporating the

19 P-cell into the forefoot region in custom molded insole, the pressure reduction was still lower than

20 that of dynamic impression insole as a result of smaller contact area. These could explain the

21 dynamic impression insole was superior to custom molded insoles in pain relief. Since RA subjects

22 with metatarsal pain have 20-40\% lower pain pressure threshold (Hodge et al., 2009), these patients

23 require more plantar pressure reduction to diminish their pain sensation.

24 Previous studies have reported $11.3 \%$ to $21.8 \%$ of pressure reductions at the MTH after using 
1 custom molded insoles (Hodge et al., 1999; Jackson et al., 2004). The magnitudes of these

2 reductions were lower than the reductions (46.3\%) made by the dynamic impression insole. Some

3 other studies reported that a custom molded insole could not reduce plantar pressure (Novak et al.,

4 2009) and did not bring any clear benefits to patients with foot pain (Conrad et al., 1996; Novak et

5 al., 2009). Different designs and materials used in the insoles leading to different ability to reduce

6 plantar pressure could explain these conflicted results.

7 Although the dynamic impression insole requires the persistent walking compression in a period

8 of time, it is very simple and low cost in orthotic fabrication. Since all the layers are adhered

9 together with double-sided adhesive tape, it is easy to adjust or renew for extending their effective

10 life to one-year or longer. It is extremely important that no organic solvent will be evaporated in all

11 the procedures. Furthermore, the metatarsalgia due to multiple forefoot deformities is very

12 individualized in subjects with rheumatoid foot. The dynamic impression insole can be customized

13 to accommodate plantar contour by their own impression. The pressure from the patient's weight and

14 dynamic forces in walking could optimize the shape of the multilayer insoles to reduce plantar

15 pressure by increasing contact area and force-absorbing capacity after sequential padding and

16 dynamic impression. In addition, metatarsal padding could further reduce metatarsal pressure by

17 redistributing force loading from the MTH to the proximal area without a pressure discomfort.

18 In fabricating custom molded insoles, a plantar mold is taken from a static impression when the

19 foot is put in a neutral subtalar and horizontal forefoot position. However, the metatarsophalangeal

20 joint is situated at a dorsiflexion position and experiences higher pressure than the static state during

21 heel-rise of the gait. Thus, the plantar mold taken from the static impression is incompatible to the

22 dynamic shape of the forefoot in walking. This could explain that custom molded insole has a less

23 contact area and higher pressure at the MTH than dynamic impression insole. In orthotic

24 management of metatarsal pain, the majority of strategies are to shift the excessive MTH force 
1 loading with a metatarsal support proximal to MTH (Hodge et al., 1999; Jackson et al., 2004). The

2 metatarsal pad utilizes only a small area to shift the excessive force from the MTH to the metatarsal

3 shaft, leading to a decrease in mean force without obvious increase in contact area. In contrast, the

4 dynamic impression insole utilizes all available area around the MTH to reduce the pressure at the

5 painful plantar areas, which corresponds to the deeper indentations on the top surface of insole

6 (figure 2). It also explained why the MTH pressure reduction in dynamic impression insole was

7 twice as those reported in previous studies for custom insoles with metatarsal support (Postema et al.,

8 1998; Hodge et al., 1999).

9 At the midfoot, the mean force and contact area were simultaneously increased in the dynamic

10 impression insoles, resulting in no significant differences in plantar pressure among the three

11 different insoles. The results were compatible with our clinical observations that all participants

12 experienced effective pain relief under their MTH without any discomfort or pain at the midfoot

13 when using the dynamic impression insole. Patients with rheumatoid feet may suffer from

14 progressive flattening or collapse of the plantar arch due to tarsal joints involvement (Bouysset et

15 al.,1987). The dynamic impression insole increased the mean force at the midfoot by 2.6 times and

16 the weight-bearing area by 2.0 times. However, as this force is only supportive rather than corrective

17 due to the compressibility of the insole, it is not recommended for RA subjects with flexible flat foot.

18 The dynamic impression insole had a more flattened arch contour than the custom molded insole

19 (figure 1). This explained why the custom molded insole has caused discomfort under the midfoot in

20 some participants, but the discomfort was not seen in patients using the dynamic impression insole.

21 The discomfort at the midfoot was similar to the initial discomfort caused by wearing the rigid

22 molded orthoses (Woodburn et al., 2002). This was resulted from a force resisting the downward

23 movement of longitudinal plantar arch at the midfoot in the stance phase of walking (Hunt et al.,

24 2001). It is interesting that the dynamic impression insole has a significantly greater mean force but 
1 a lower pressure at the midfoot compared to the custom molded insole.

2 At the midfoot, the mean force and contact area were simultaneously increased in both custom

3 molded and dynamic impression insoles, resulting in no significant differences in plantar pressure

4 among the three different insoles. The results were compatible with our clinical observations that all

5 participants experienced effective pain relief under their MTH without any discomfort or pain at the

6 midfoot when using the dynamic impression insole. Patients with rheumatoid feet may suffer from

7 progressive flattening or collapse of the plantar arch due to tarsal joints involvement (Bouysset et

8 al.,1987). The dynamic impression insole could also provide an arch support with 2.6 times the mean

9 force and 2.0 times the weight-bearing area at the midfoot. Since the force is only supportive rather

10 than corrective, the insole is not recommended for RA subjects with flexible flat foot. Similar to the

11 initial discomfort caused by wearing the rigid molded orthoses (Woodburn et al., 2002), our custom

12 molded insole also caused a plantar discomfort under the midfoot in some participants. The pressure

13 discomfort was resulted from a rigid force to resist the downward movement of longitudinal plantar

14 arch at midfoot in the stance phase of walking (Hunt et al., 2001). This downward movement of

15 plantar arch could also explain that dynamic impression insole had a more flattened arch contour

16 than custom molded insole in the study (figure 1). It is interesting that the dynamic impression insole

17 has a greater mean force but a lower pressure discomfort at the midfoot compared to the custom

18 molded insole.

19 The dynamic impression insole possessed a superior pressure reduction due to a larger

20 weight-bearing area at the MTH compared to the custom molded insole, but no significant

21 differences were found in all pressure-variables at the heel between two insoles. These findings

22 indicate that custom-made insoles will be same effective in heel pressure reduction regardless of

23 using static impression or dynamic impression. This hypothesis is supported by the fact that tarsal

24 joints are in a relatively stationary status in walking as compared to the most mobile 
1 metatarsophalangeal joints. The foot joints motion in gait cycle could also explain that dynamic

2 impression insole had a greater contact area than custom molded insole only at the MTH, but no

3 significant differences at the heel, midfoot and toes.

\section{Conclusions}

In the custom molded and dynamic impression insoles, the MTH pressure was reduced due to the increase of contact area with a concomitant decrease of loading at the forefoot. However, the dynamic impression insole is superior to custom molded insole to reduce pressure because it

9 provides a larger weigh-bearing area from an optimal forefoot contouring on the insole. In contrast to the less contour change at the heel during walking, the dynamic status of forefoot is an important

11 factor that should be taken into consideration in orthotic designs for reducing the MTH pressure. In orthotic management of metatarsal pain, we recommend using the materials with memory

13 properties to dynamically accommodate the painful MTH for maximum pressure reduction. Since this study is a preliminary evaluation in repeated measures design, further investigations can be carried out on a large scale for randomized controlled trials.

\section{References}

18 Arnett, F.C., Edworthy, S.M., Bloch, D.A., McShane, D.J., Fries, J.F., Cooper, N.S., Healey,

19 L.A., Kaplan, S.R., Liang, M.H., Luthra, H.S., 1988. The American Rheumatism Association

Bertram, G., Hsu, J.D., 1997. AAOS Atlas of orthoses and assistive device: Orthotic management of 1987 revised criteria for the classification of rheumatoid arthritis. Arthritis Rheum 31, 315-324. neuropathic and dysvascular patient. Third ed. Mosby Incorp. St. Louis, MO, pp. 442-444.

24 tarsal arthritis on X-ray. Scandinavian Journal of Rheumatology 16, 127-133. 
1 Chalmers, A.C., Busby, C., Goyert, J., Porter, B., Schulzer, M., 2000. Metatarsalgia and rheumatoid

2 arthritis : A randomized, single blind, sequential trial comparing 2 types of foot orthoses and

3 supportive shoes. The Journal of Rheumatology 27,1643-1647.

4 Clark, H., Rome, K., Plant, M., O’Harey, K., Gray, J., 2006. A critical review of foot orthoses in the 5 rheumatoid arthritic foot. Rheumatology 45,139-145.

6 Conrad, K., Budiman-Mak, E., Roach, K.E., Hedeker, D., 1996. Impacts of foot orthoses on pain and 7 disability in rheumatoid arthritis. Journal of Clinical Epidemiology 49, 1-7.

8 Fauci, A.S., Braunwald, E., Kasper, D.L., Hauser, S.L., Longo, D.L., Jameson, J.L., Loscalzo, J., 9 2008. Harrison's principles of internal medicine 17th ed. McGraw-Hill Incorp, New York, pp. 102083.

11 Faul, F., Erdfelder, E., Lang, A-G., Buchner, A., 2007. G*Power 3: A flexible statistical power analysis program for the social, behavioral, and biomedical sciences. Behavior Research Methods 39, 175-191.

Hodge, M.C., Bach, T.M., Carter, G.M., 1999. Orthotic management of plantar pressure and pain in rheumatoid arthritis. Clinical Biomechanics 14, 567-575.

Hodge, M.C., Nathan, D., Bach, T.M., 2009. Plantar pressure pain thresholds and touch sensitivity in rheumatoid arthritis. Foot and Ankle International 30, 1-9.

Hunt, A.E., Smith, R.M., Torode, M., Keenan, AM., 2001. Inter-segment foot motion and ground reaction forces over the stance phase of walking. Clinical Biomechanics 16, 592-600

Jackson, L., Binning, J., Potter, J., 2004. Plantar pressures in rheumatoid arthritis using prefabricated metatarsal padding, Journal of the American Podiatric Medical Association, 94, 239-245.

Jeng, C., Campbell, J., 2008. Current concepts review: the rheumatoid forefoot. Foot and Ankle International 29, 959-968.

24 Kang, H.G., Dingwell, J.B., 2008. Separating the effects of age and walking speed on gait variability, 
Gait and Posture 27, 572-577.

2 Kerry, R.M., Holt, G.M., Stockley, I., 1994. The foot in chronic rheumatoid arthritis: a continuing

3 problem. The Foot 4, 201-203.

4 MacSween, A., Brydson, G., Hamilton, J., 1999. The effect of custom moulded ethyl vinyl acetate

5 foot orthoses on the gait on patients with in rheumatoid arthritis. The Foot 9, 128-133.

6 Novak, P., Burger, H., Tomsic, M., Marincek, C., 2009. Influence of foot orthoses on plantar

7 pressures, foot pain and walking ability of rheumatoid arthritis patient - a randomised controlled

8 study. Disability and Rehabilitation. 31, 638-645.

9 Otter, S.J., Bowen, C.J., Young, A.K., 2004. Forefoot plantar pressures in rheumatoid arthritis.

10 Journal of the American Podiatric Medical Association 94, 255-260.

11 Postema. K., Burm, P.E.T., van der Zande, M.E., van Limbeek, J., 1998. Primary metatarsalgia: the influence of a custom moulded insole and a rockerbar on plantar pressure. Prosthetics and Orthotics International 22, 35-44.

Speigel, T.M., Spiegel, J.S., 1982. Rheumatoid arthritis in the foot and ankle - diagnosis, pathology

17 Sumpio, B.E., 2000. Foot ulcers. New England Journal of Medicine 343, 787-793.

18 Vainio, K., 1975. Orthopaedic surgery in the treatment of rheumatoid arthritis. Annals of Clinical $19 \quad$ Research 7, $216-224$.

20 van der Leeden, M., Steultjens, M., Dekker, J.H.M., Prins, A.P.A, Dekker, J., 2006. Forefoot joint 21 damage, pain and disability in rheumatoid arthritis patients with foot complaints: the role of 22 plantar pressure and gait characteristics. Rheumatology 45, 465-469.

23 Woodburn, J., Barker, S., Helliwell, P.S., 2002. A randomized Controlled Trial of Foot Orthoses in 
Rheumatoid Arthritis. The Journal of Rheumatology 29, 1377-1383.

2 Zhu, H., Wertsch, J.J., Harris, G.F., Alba, H.M., 1995. Walking cadence effect on plantar pressures.

3 Archives of Physical Medicine and Rehabilitation 76, 1000-1005.

4 
1 Table 1

2 Demographic characteristics of subjects $(n=17)$

\begin{tabular}{lcc}
\hline Characteristics & Mean (SD) & Range \\
\hline Age (years) & $58.6(10.1)$ & $42-74$ \\
Body weight $(\mathrm{Kg})$ & $54.6(9.9)$ & $35-73$ \\
Body height $(\mathrm{cm})$ & $155.6(6.7)$ & $143-168$ \\
Body mass $\left(\mathrm{Kg} / \mathrm{m}^{2}\right)$ & $22.5(3.6)$ & $14.6-28.9$ \\
Disease duration (years` & $16.3(7.1)$ & $6-32$ \\
\hline
\end{tabular}

3

4

5 
1 Table 2

2 Peak pressure $(\mathrm{kPa})$ for three different insoles $(\mathrm{n}=17)$

\begin{tabular}{lccccc}
\hline Plantar regions & 7-mm flat EVA & Custom & \multicolumn{2}{c}{ Dynamic } & \multicolumn{2}{c}{ ANOVA } \\
& (40 Shore A) & molded insole & impression insole & F value & p value \\
\hline \multirow{2}{*}{ Heel } & $238.8(50.8)$ & $138.9(30.0)$ & $146.5(28.1)$ & \multirow{2}{*}{61.9} & $<0.001^{*}$ \\
\multirow{4}{*}{ Midfoot } & $0 \%$ & $-41.8 \% * *$ & $-38.6 \% * *$ & & \\
& $108.1(40.7)$ & $102.9(22.7)$ & $95.2(18.9)$ & 2.7 & 0.097 \\
MTH & $0 \%$ & $-4.8 \%$ & $-11.9 \%$ & & \\
& $275.5(57.6)$ & $180.9(51.4)$ & $147.9(32.0)$ & 144.3 & $<0.001^{*}$ \\
Toes & $0 \%$ & $-34.4 \% * *$ & $-46.3 \% * *^{\dagger}$ & & \\
& $174.3(71.3)$ & $147.9(59.8)$ & $147.9(44.8)$ & 3.1 & 0.074 \\
\hline
\end{tabular}

3 Values are expressed as mean (standard deviation) and \% change of mean values normalized to those of 4 EVA,

$5 *=P<0.05$, compared among three insoles using one-way ANOVA with repeated measures,

$6 * *=P<0.05$, dynamic impression insole and custom molded insole compared to 7-mm flat EVA,

$7{ }^{\dagger}=P<0.05$, compared between dynamic impression insole and custom molded insole. 
1 Table 3

2 Pressure-time integrals (kPa s) for three different insoles $(\mathrm{n}=17)$

\begin{tabular}{lccccc}
\hline Plantar regions & 7-mm flat EVA & Custom & Dynamic & \multicolumn{2}{c}{ ANOVA } \\
& (40 Shore A) & molded insole & impression insole & F value & p value \\
\hline \multirow{2}{*}{ Heel } & $79.6(17.4)$ & $48.0(11.0)$ & $49.1(11.0)$ & 43.7 & $<0.001^{*}$ \\
& $0 \%$ & $-39.7 \% * *$ & $-38.3 \%^{* *}$ & & \\
\multirow{2}{*}{ Midfoot } & $43.7(18.6)$ & $45.0(11.8)$ & $44.2(11.0)$ & 0.1 & 0.909 \\
& $0 \%$ & $2.9 \%$ & $1.1 \%$ & & \\
MTH & $88.7(21.4)$ & $56.8(16.1)$ & $45.3(11.8)$ & 86.0 & $<0.001^{*}$ \\
& $0 \%$ & $-36.0 \% * *$ & $-48.9 \%^{* *}$ & & \\
Toes & $50.0(26.5)$ & $45.8(23.0)$ & $41.1(16.1)$ & 2.6 & 0.109 \\
\hline
\end{tabular}

3 Values are expressed as mean (standard deviation) and \% change of mean values normalized to those of 4 EVA,

$5 *=P<0.05$, compared among three insoles using one-way ANOVA with repeated measures,

$6 * *=P<0.05$, dynamic impression insole and custom molded insole compared to 7-mm flat EVA,

$7{ }^{\dagger}=P<0.05$, compared between dynamic impression insole and custom molded insole. 
2 Table 4

3 Mean force (Newton) for three different insoles ( $\mathrm{n}=17)$

\begin{tabular}{lccccc}
\hline Plantar regions & 7-mm flat EVA & Custom & \multicolumn{2}{c}{ Dynamic } & \multicolumn{2}{c}{ ANOVA } \\
& $(40$ Shore A) & molded insole & impression insole & F value & p value \\
\hline \multirow{2}{*}{ Heel } & $168.9(33.5)$ & $131.6(33.5)$ & $129.2(32.7)$ & 45.2 & $<0.001^{*}$ \\
& $0 \%$ & $-22.1 \%^{* *}$ & $-23.5 \%^{* *}$ & & \\
\multirow{2}{*}{ Midfoot } & $42.4(33.1)$ & $89.8(43.0)$ & $110.6(43.4)$ & 83.2 & $<0.001^{*}$ \\
\multirow{2}{*}{ MTH } & $0 \%$ & $111 . \%^{* *}$ & $160.7 \%^{* *}$ & & \\
& $162.9(63.8)$ & $131.2(57.3)$ & $133.4(50.6)$ & 21.0 & $<0.001^{*}$ \\
\multirow{2}{*}{ Toes } & $0 \%$ & $-19.5 \%^{* *}$ & $-18.1 \%^{* *}$ & & \\
& $26.2(14.0)$ & $37.4(19.0)$ & $36.0(16.4)$ & 9.7 & $0.002^{*}$ \\
\hline
\end{tabular}

4 Values are expressed as mean (standard deviation) and \% change of mean values normalized to those of 5 EVA,

$6 *=P<0.05$, compared among three insoles using one-way ANOVA with repeated measures,

$7 \quad * *=P<0.05$, dynamic impression insole and custom molded insole compared to 7 -mm flat EVA,

$8{ }^{\dagger}=P<0.05$, compared between dynamic impression insole and custom molded insole. 
1 Table 5

2 Contact area $\left(\mathrm{cm}^{2}\right)$ for three different insoles $(\mathrm{n}=17)$

\begin{tabular}{lccccc}
\hline Plantar regions & 7-mm flat EVA & Custom & Dynamic & \multicolumn{2}{c}{ ANOVA } \\
& (40 Shore A) & molded insole & impression insole & F value & p value \\
\hline \multirow{2}{*}{ Heel } & $33.0(5.4)$ & $37.0(5.4)$ & $35.7(5.0)$ & 30.6 & $<0.001^{*}$ \\
\multirow{2}{*}{ Midfoot } & $0 \%$ & $12.1 \% * *$ & $8.2 \%^{* *}$ & & \\
& $22.1(11.9)$ & $40.8(10.5)$ & $44.7(7.3)$ & 48.6 & $<0.001^{*}$ \\
MTH & $0 \%$ & $84.6 \%^{* *}$ & $102.5 \%^{* *}$ & & \\
& $38.5(7.7)$ & $41.0(8.3)$ & $44.3(6.3)$ & 21.5 & $<0.001^{*}$ \\
Toes & $0 \%$ & $6.5 \% * *$ & $15.1 \%^{* *}$ & & \\
& $11.7(4.9)$ & $15.1(5.9)$ & $15.3(4.7)$ & 13.8 & $0.001^{*}$ \\
\hline
\end{tabular}

3 Values are expressed as mean (standard deviation) and \% change of mean values normalized to those of 4 EVA,

$5 *=P<0.05$, compared among three insoles using one-way ANOVA with repeated measures,

$6 * *=P<0.05$, dynamic impression insole and custom molded insole compared to 7-mm flat EVA,

$7{ }^{\dagger}=P<0.05$, compared between dynamic impression insole and custom molded insole. 